\title{
NOUVELLE
}

\section{Vers un vaccin prophylactique bivalent contre les virus des hépatites $B$ et $C$ ?}

Élodie Beaumont, Philippe Roingeard

Inserm U966, Université François Rabelais et CHRU de Tours, 10, boulevard Tonnellé 37032 Tours, France.

elodie.beaumont@univ-tours.fr

philippe.roingeard@univ-tours.fr

\section{L'hépatite C en 2013}

Au cours des dernières années, des progrès majeurs ont été réalisés en termes de traitements contre le virus de l'hépatite $C$ (VHC), avec la mise au point d'antiviraux à action directe (DAA, pour direct-acting antiviral agents), notamment des inhibiteurs de la protéase virale $[1,14](\rightarrow)$.

$\rightarrow$ Voir la Synthèse de S. Pol, $\mathrm{m} / \mathrm{s}$ novembre 2013, $n^{\circ} 11$, page 998

Cependant, ces traitements sont très coûteux, souvent associés à d'importants effets secondaires, et ne suffisent donc pas à traiter les 170 millions de personnes chroniquement infectées par le VHC dans le monde. Par ailleurs, l'organisation mondiale de la santé (OMS) estime que près de 4 millions de nouvelles infections par le VHC surviennent chaque année dans le monde. Ce problème de santé publique n'est pas confiné aux pays en développement puisqu'on estime à environ 18000 le nombre de nouvelles infections tous les ans aux États-Unis, soit une toutes les trente minutes [2]. Comme la plupart de ces nouvelles infections sont asymptomatiques, les personnes infectées ignorent le plus souvent qu'elles deviennent porteuses chroniques du virus. Elles ne sont donc pas prises en charge médicalement et sont par ailleurs susceptibles de transmettre le virus à des sujets sains. Si l'infection par le VHC n'est pas détectée lors d'un examen de médecine préventive, la maladie ne se révèle que plusieurs années après l'infection, bien souvent sous la forme d'une hépatite chronique active ayant induit des lésions hépatiques. À ce stade, les traitements antiviraux sont toutefois beaucoup moins efficaces. Pour toutes ces raisons, la mise au point d'un vaccin prophylactique contre le VHC est un enjeu majeur. Un tel vaccin représente le meilleur espoir de pouvoir contrôler l'épidémie à l'échelle mondiale, ainsi qu'une opportunité de diminuer considérablement les dépenses de santé liées aux traitements des infections chroniques par le VHC.

Les potentiels vaccins prophylactiques contre le VHC

L'infection par le VHC peut être spontanément résolutive chez certains sujets récemment contaminés, ainsi que chez le chimpanzé, la seule espèce animale pouvant être infectée expérimentalement par ce virus. Ce phénomène fournit des arguments solides pour le développement d'un vaccin préventif contre le VHC. Dans le même temps, la complexité de la mise au point d'un tel vaccin est soulignée par la persistance fréquente du virus en raison de sa capacité à muter pour échapper à l'immunité humorale et cellulaire [3]. Dans ce contexte, deux grands types de vaccins prophylactiques ont été proposés [4] : ceux basés sur l'induction d'une réponse humorale contre les protéines d'enveloppe du virus, destinée à neutraliser ce dernier; et ceux visant à induire une réponse cellulaire contre la protéine de capside ou les protéines non structurales du virus, qui sont très conservées au sein des différents génotypes du VHC. Les réponses immunitaires déclenchées par ces deux types de vaccin sont ainsi complémentaires et ceux-ci pourraient d'ailleurs être associés pour obtenir une protection optimale. Évalués dans un modèle chim- panzé, ces vaccins ont donné des résultats intéressants, bien que ne protégeant pas complètement de l'infection expérimentale occasionnée chez les animaux immunisés [5]. Si dans certains cas ils empêchaient l'infection, ils pouvaient au moins, dans les autres cas, diminuer de façon importante la virémie et enrayer le développement d'une infection chronique. Deux candidats vaccins ayant généré des résultats prometteurs dans un modèle chimpanzé ont fait l'objet d'essais de phase I chez l'homme. Un premier vaccin, basé sur l'usage de protéines d'enveloppes recombinantes $\varepsilon l$ et ६2 du VHC purifiées, s'est avéré capable d'induire une bonne réponse humorale chez l'homme, permettant la neutralisation de différents génotypes du VHC in vitro [6]. Le second, basé sur l'injection de vecteurs adénoviraux exprimant les protéines non structurales du VHC, était capable d'induire une bonne réponse cellulaire multi-épitopique [7]. Ce dernier est actuellement en essai de phase II aux États-Unis pour évaluer la protection induite chez des toxicomanes utilisateurs de drogues par voie intraveineuse.

Les difficultés rencontrées lors de la mise au point d'un vaccin prophylactique basé sur les protéines d'enveloppe du VHC

Le concept d'un vaccin basé sur les protéines d'enveloppe $\varepsilon 1$ et $\varepsilon 2$ du VHC semble prometteur, mais un obstacle majeur entrave la poursuite de son développement. Ces deux protéines possèdent un domaine transmembranaire (DTM) qui les retient dans les compartiments intracellulaires, rendant leur extraction et 


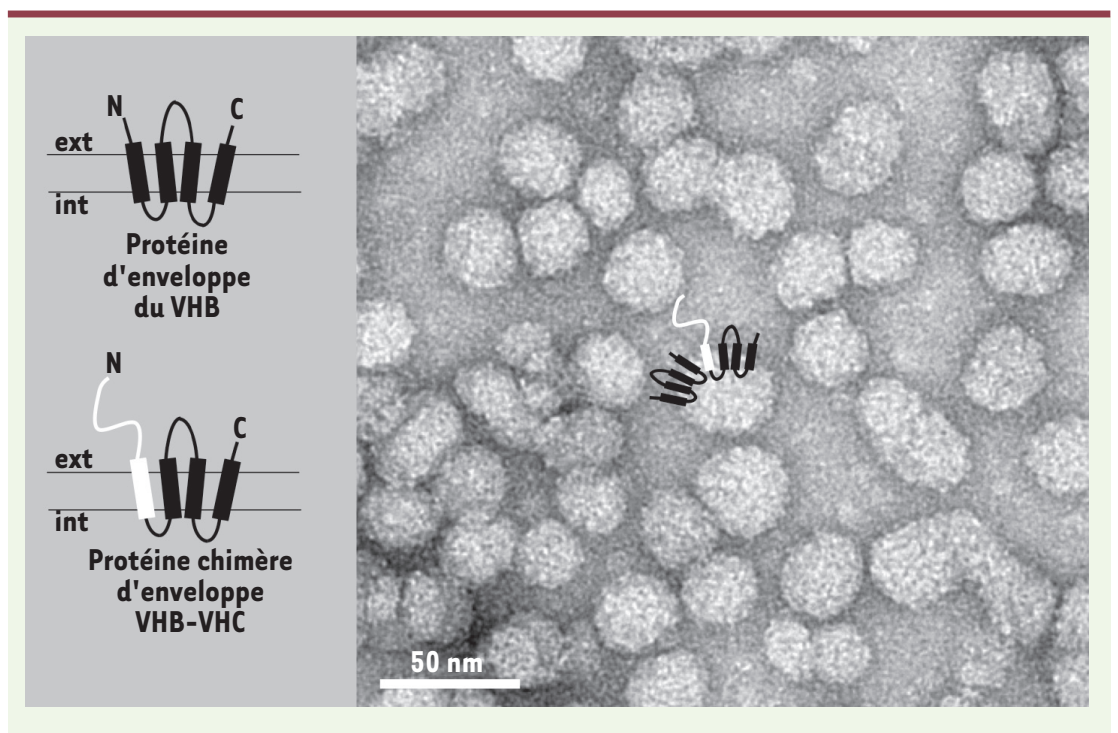

Figure 1. Principe des particules chimères d'enveloppe VHB-VHC. La protéine d'enveloppe $S$ (pour surface) du virus de l'hépatite B (VHB) est une protéine qui présente quatre domaines transmembranaires (DTM). Exprimée dans une cellule eucaryote, cette protéine est capable de s'auto-assembler en particules sous-virales d'environ $25 \mathrm{~nm}$ de diamètre composées d'une centaine de copies de $S$. Les extrémités amino-terminales et carboxy-terminales de $S$ ainsi qu'une boucle antigénique sont exposées à la surface des particules. Ces particules sous virales sont très immunogènes et constituent la base du vaccin contre le VHB. Les deux protéines d'enveloppe $\varepsilon l$ et $\varepsilon 2$ du virus de l'hépatite C (VHC) présentent un seul DTM qui est essentiel à leur conformation et leur immunogénicité. Des protéines chimères, dans lesquelles le DTM amino-terminal de $S$ est substitué par celui des protéines $\varepsilon l$ ou $\varepsilon 2$, sont capables de s'auto-assembler avec la protéine sauvage $S$ en particules chimères d'enveloppe incorporant $\varepsilon 1$ ou $\varepsilon 2$ dans leur intégralité [13].

leur purification incompatibles avec un développement industriel. Ces difficultés ont conduit à l'élaboration de stratégies alternatives basées sur l'utilisation de protéines $\varepsilon 1$ et/ou $\varepsilon 2$ tronquées, pouvant être sécrétées. Toutefois, ces approches ont rencontré un succès limité, puisque la suppression de leur DTM diminue considérablement leurs propriétés antigéniques et fonctionnelles [8]. Plus récemment, des particules rétrovirales murines pseudotypées pour les protéines d'enveloppe du VHC ont été proposées comme une nouvelle plate-forme de vaccination [9]. Ces particules appelées VHCpp (pour pseudoparticules) présentent les protéines $\varepsilon 1$ et $\varepsilon 2$ dans une conformation correcte et sont couramment utilisées comme modèle d'étude de l'entrée du VHC. Cependant, leur production à grande échelle s'avère complexe, et l'utilisation de particules rétrovirales animales pour le dévelop- patite $B(V H B)$, fondé sur la propriété de sa protéine majeure d'enveloppe (la protéine $S$, pour surface, présentant quatre DTM) à s'auto-assembler en petites particules sous-virales non infectieuses (Figure 1). Ces particules, constituées d'une centaine de copies de la protéine $S$, sont très immunogènes et représentent la base du vaccin contre le VHB, utilisé avec succès depuis le début des années 1980. Plusieurs approches vaccinales originales visant à incorporer des épitopes hétérologues en positions amino- ou carboxy-terminales de la protéine $S$ du VHB ont depuis été développées [12]. Le DTM des protéines $\varepsilon 1$ et દ2 du VHC étant essentiel à leur conformation et leur immunogénicité, nous avons proposé récemment une stratégie innovante en élaborant des protéines d'enveloppe chimères pour lesquelles le DTM amino-terminal de la protéine $S$ du VHB a été substitué par le DTM des protéines $\varepsilon 1$ ou $\varepsilon 2$ du VHC [13] (Figure 1). Exprimées dans la lignée $\mathrm{CHO}$ (chinese hamster ovary cells, une lignée approuvée pour les biomédicaments), ces protéines d'enveloppe chimères VHB-VHC sont co-assemblées avec la protéine sauvage $S$ en particules sousvirales, qui sont efficacement sécrétées (Figure 1). Ces particules ressemblent à celles du vaccin contre le VHB et présentent l'avantage d'arborer les protéines d'enveloppe du VHC dans leur intégralité. Elles peuvent induire dans un modèle animal la production d'anticorps qui neutralisent in vitro différents génotypes du VHC. En effet, bien que les particules utilisées lors des premiers essais d'immunisation contenaient une enveloppe de VHC de génotype la, tous les génotypes testés ont été neutralisés par les anticorps induits, même si cette neutralisation se faisait de manière plus efficace pour les génotypes la et lb que pour les génotypes $2 a$ et $3 a$, plus distants génétiquement [13]. Par ailleurs, ces particules chimères induisent une réponse équivalente à celle induite par un vaccin commercial contre le VHB, renforçant ainsi l'idée que de telles par- 
ticules vaccinales puissent se substituer aux vaccins contre le VHB, avec l'avantage de pouvoir aussi protéger contre le VHC. Ces particules présentent également l'avantage de pouvoir être produites selon les procédures établies pour le vaccin contre le VHB, ce qui réduirait les délais et coûts de développement industriel. Tous ces résultats obtenus en modèle animal nécessitent d'être confirmés chez l'homme, mais indiquent néanmoins que la mise au point d'un vaccin «bivalent», qui protégerait de l'infection par ces deux agents majeurs des hépatites virales humaines, représente une éventualité crédible. $\diamond$

Towards a bivalent prophylactic vaccine against hepatitis $B$ and $C$ viruses?

\section{REMERCIEMENTS}

Nos travaux sur le développement d'un vaccin bivalent VHB-VHC ont été soutenus par l'Institut Mérieux, le programme FEDER (Fonds euro- péens de développement régional) et le Cluster de recherche en infectiologie de la région centre. Ils sont soutenus actuellement par l'ANR (grant Emergence Hepatibivax) et l'ANRS.

\section{LIENS D'INTÉRÊT}

Les auteurs déclarent n'avoir aucun lien d'intérêt concernant les données publiées dans cet article.

\section{RéFÉRENCES}

1. Sarrazin C, Hézode C, Zeuzem S, Pawlotsky JM. Antiviral strategies in hepatitis C virus infection.) Hepatol 2012 ; 56 : S88-100.

2. Edlin BR. Perspective: test and treat this silent killer. Nature 2011 ; 474 : S18-9.

3. Rehermann B. Hepatitis C virus versus innate and adaptive immune responses: a tale of coevolution and coexistence. J Clin Invest 2009 ; 119 : 1745-54.

4. Houghton M. Prospects for prophylactic and therapeutic vaccines against the hepatitis $C$ viruses. Immunol Rev 2011 ; 239 : 99-108.

5. Dahari H, Feinstone SM, Major ME. Meta-analysis of hepatitis $C$ virus vaccine efficacy in chimpanzees indicates an importance for structural proteins. Gastroenterology 2010 ; 139 : 965-74.

6. Stamataki Z, Coates S, Abrignani S, Houghton M, McKeating JA. Immunization of human volunteers with hepatitis $C$ virus envelope glycoproteins elicits antibodies that cross-neutralize heterologous virus strains. J Infect Dis 2011 ; 204 : 811-3.

7. Barnes $\varepsilon$, Folgori A, Capone $S$, et al. Novel adenovirusbased vaccines induce broad and sustained T cell responses to HCV in man. Sci Transl Med $2012 ; 4$ : $115 \mathrm{ral}$.

8. Sominskaya I, Alekseeva E, Skrastina D, et al. Signal sequences modulate the immunogenic performance of human hepatitis $C$ virus $\varepsilon 2$ gene. Mol Immunol $2006 ; 43: 1941-52$

9. Garrone P, Fluckiger AC, Mangeot PE, et al. A primeboost strategy using virus-like particles pseudotyped for HCV proteins triggers broadly neutralizing antibodies in macaques. Sci Transl Med 2011 ; 3 : 94 ra71.

10. Legrand-Abravanel F, Izopet J. Culture du virus de I'hépatite C, enfin! Med Sci (Paris) 2007 ; 23 : 588-9.

11. Akazawa D, Moriyama M, Yokokawa H, et al. Neutralizing antibodies induced by cell culturederived hepatitis $C$ virus protect against infection in mice. Gastroenterology 2013 ; 145 : 447-55.

12. Phogat $S$, Svehla K, Tang M, et al. Analysis of the human immunodeficiency virus type 1 gp 41 membrane proximal external region arrayed on hepatitis $B$ surface antigen particles. Virology $2008 ; 373: 72-84$

13. Beaumont $\varepsilon$, Patient R, Hourioux C, et al. Chimeric hepatitis $B$ virus/hepatitis $C$ virus envelope proteins elicit broadly neutralizing antibodies and constitute a potential bivalent prophylactic vaccine. Hepatology $2013 ; 57: 1303-13$.

14. Pol S. Virus de l'hépatite $C: 25$ ans, la fin de l'histoire? Med Sci (Paris) 2013 ; 29 : 998-1003.

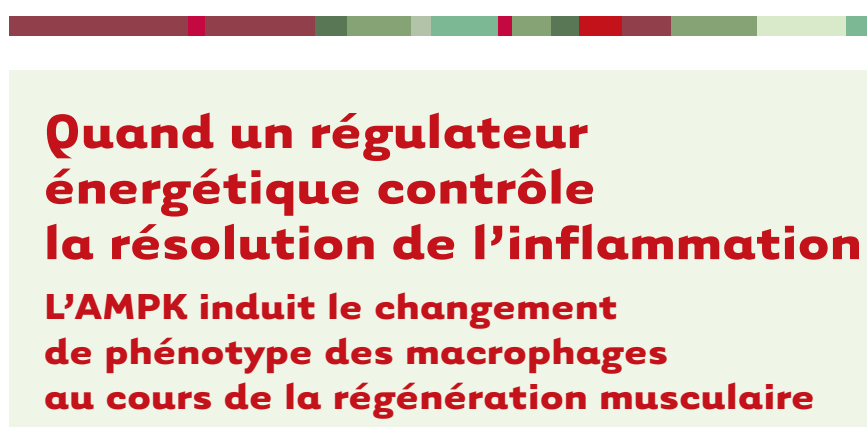

Marine Théret, Bénédicte Chazaud, Rémi Mounier
> La régénération musculaire est un processus largement étudié depuis de nombreuses années. Cette capacité de réparation du muscle est rendue possible notamment grâce aux cellules souches musculaires adultes, appelées cellules satellites, présentes entre la lame basale et la fibre musculaire [1]. À l'état basal, ces cellules sont quiescentes. Lors d'une lésion, elles sont activées et prolifèrent pour former une population de myoblastes qui, dans un second temps, soit s'engagent dans la voie de la myogenèse - pour devenir myocytes et fusionner pour former de nouvelles fibres remplaçant les fibres musculaires lésées -, soit retournent dans un état de quiescence pour former le réservoir de cellules satellites (revue dans [1]). La coordination de ces événements est finement régulée au niveau de la cellule musculaire ellemême, mais également par son environnement tissulaire. En effet, différents types cellulaires sont nécessaires à une
Inserm, U1016, Institut Cochin, 24, rue du Faubourg Saint-Jacques, 75014 Paris, France ; CNRS, UMR8104, 75014 Paris, France ; Université Paris Descartes, Sorbonne Paris Cité, 75014 Paris, France.

remi.mounier@inserm.fr benedicte.chazaud@inserm.fr

bonne régénération musculaire, dont les macrophages [2].

Les macrophages : cellules immunitaires indispensables à la régénération musculaire Les macrophages, principalement connus comme l'une des premières défenses de l'organisme face à une infection, ont aussi d'autres rôles. Leur participation à la réparation de nombreux tissus a ainsi été mise en évidence ces dernières 